Musique et Sciences Sociales

Hors-série 1 | 2018

Musique, histoire, sociétés

\title{
Musique, histoire et société
}

Les études sur la musique à l'EHESS

Talia Bachir-Loopuyt et Igor Contreras Zubillaga

\section{OpenEdition}

\section{Journals}

Édition électronique

URL : http://journals.openedition.org/transposition/1812

DOI : 10.4000/transposition. 1812

ISSN : 2110-6134

Éditeur

CRAL - Centre de recherche sur les arts et le langage

Référence électronique

Talia Bachir-Loopuyt et Igor Contreras Zubillaga, « Musique, histoire et société », Transposition [En ligne], Hors-série 1 | 2018, mis en ligne le 09 mai 2018, consulté le 09 février 2020. URL : http:// journals.openedition.org/transposition/1812 ; DOI : 10.4000/transposition.1812

Ce document a été généré automatiquement le 9 février 2020

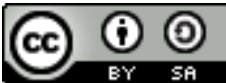

La revue Transposition est mise à disposition selon les termes de la Licence Creative Commons Attribution - Partage dans les Mêmes Conditions 4.0 International. 


\title{
Musique, histoire et société
}

\author{
Les études sur la musique à l'EHESS \\ Talia Bachir-Loopuyt et Igor Contreras Zubillaga
}

1 Les numéros de la revue Transposition. Musique et sciences sociales nous ont habitués au rythme cyclique d'appels et de parutions sur des thématiques circonscrites. Ce numéro hors-série est le fruit d'un travail mené sur un temps à la fois plus long et plus éclaté. Initialement prévu pour les 40 ans de l'EHESS (en 2015) puis pour les 70 ans de la VI section de l'EPHE (2017), il paraît finalement en 2018 alors que les commémorations d'autres anniversaires battent leur plein ${ }^{1}$. Il conserve cependant l'objectif initial d'un retour sur le terreau institutionnel et intellectuel dont sont issus les coordinateurs de ce numéro ${ }^{2}$ et la plupart des membres du comité de rédaction de la revue Transposition: l'École des Hautes Études en Sciences Sociales et, pour beaucoup, la formation doctorale «Musique, Histoire, Société » qui a vu le jour au sein de cette institution au début des années $2000^{3}$.

Reconnaissons-le, avec les auteurs de ce numéro : ceux qui sont passés à un moment de leur parcours par l'EHESS ou ceux qui y sont encore aujourd'hui actifs comme enseignants-chercheurs, directeurs d'études, maîtres de conférences, membres associés de laboratoires ou doctorants ont tous acquis l'idée que cette institution constitue un monde à part dans le champ des sciences humaines et sociales en France et, peut-être plus encore, dans celui des études sur la musique. Cette singularité, qu'une rhétorique commune valorisant "l'excellence», «l'interdisciplinarité » ou "l'ouverture à l'international » peine à entièrement capturer, tient à la présence au sein de l'institution de figures et travaux qui ont fait date dans l'histoire des sciences humaines et sociales ${ }^{4}$ et les études sur les arts ${ }^{5}$. Elle tient aussi au sentiment de socialité partagée ${ }^{6}$ que procurent la fréquentation de séminaires et la familiarisation avec des travaux, notions et outils forgés «à l'école» désormais entrés dans le langage quotidien de ses chercheurs et étudiants ${ }^{7}$.

3 Pour autant, l'EHESS et, avant elle, la VI ection de l'EPHE, n'ont jamais été des îles dans l'histoire des sciences humaines et sociales. Elles n'ont pas non plus constitué des lieux de savoir unifiés. Cette complexité ressort tout particulièrement lorsque l'on entreprend de réfléchir à la place des recherches et enseignements sur la musique dans 
le périmètre de cette institution depuis sa fondation - ou ses refondations successives : 1947 pour la VI e section de l'EPHE, 1975 pour l'EHESS. Pas plus hier qu'aujourd'hui, les études sur la musique menées par des chercheurs de l'EHESS, affiliés aux laboratoires ou aux institutions partenaires, ne peuvent être appréhendées comme le reflet d'une école de pensée. Elles ne se prêtent pas non plus à une vision englobante ordonnant les divers secteurs et domaines de compétence au sein d'un espace disciplinaire cadastré ("une musicologie EHESS»), mais recouvrent des démarches de connaissance ${ }^{8}$ plurielles, pour une part contradictoires, dont l'un des seuls aspects récurrents est peut-être qu'elles se définissent comme distinctes de ce que l'on appellera parfois, une «musicologie traditionnelle». Encore faut-il définir ce que cette expression recouvre, dans le contexte français ${ }^{9}$ ou dans d'autres (musicology, Musikwissenschaft...), et avoir à l'esprit que cette frontière entre «musicologie traditionnelle " et «nouvelles musicologies » est, selon les lieux et personnes qui en font usage, employée pour signifier des différences variables. Elle tend aussi à masquer une histoire ancienne et complexe de contacts entre divers savoirs prenant la musique pour objet dans le périmètre des sciences humaines et sociales : contacts qui ont parfois donné lieu à l'institutionnalisation de disciplines «hybrides» (comme l'ethnomusicologie) mais aussi à des démarches de connaissance plurielles (sociologies, anthropologies, histoires de la musique, etc. ${ }^{10}$ ), à des "rendez-vous manqués " mais aussi à des croisements fructueux renouvelant les conceptions et pratiques l'histoire, de la sociologie, de l'anthropologie, de la philosophie - pour ne citer que ces domaines. Les contributions rassemblées dans ce numéro font de même apparaître des cheminements singuliers qui puisent dans divers savoirs scientifiques, y compris dans les outils élaborés au sein de l'espace éclectique des sciences de la musique (l'histoire de la musique, l'analyse musicale, l'ethnomusicologie, la sociologie de la musique, l'informatique, la psychologie, l'acoustique...). Ajoutons enfin que, comme d'autres démarches élaborées dans les réseaux institutionnels de la musicologie, elles se nourrissent aussi largement d'un dialogue mené, au-delà du champ scientifique, avec des praticiens des mondes de la musique.

4 Tout comme les départements de musicologie établis au sein d'universités et en dépit de différences certaines sur le plan de l'organisation institutionnelle de la production des savoirs, l'EHESS n'échappe pas aux questionnements que peut soulever l'autonomisation d'un secteur de recherche et d'enseignement dévolu à la musique. C'est là un des paradoxes - qui en fait en même temps la richesse - des programmes de formation qui se sont établis depuis le début des années 2000 dans cette école, et en particulier dans son pôle parisien, autour de l'objet «musique». Ces programmes se sont appuyés sur une pluralité de dynamiques collectives et individuelles de réflexion ${ }^{11}$ dont certaines questionnaient la pertinence même d'une mise en exergue de l'objet «musique » au sein des sciences sociales. Par là, ils ont permis de rebattre les cartes de l'équilibre entre histoire, anthropologie et sociologie (au profit de ces deux dernières, relativement peu représentées dans le champ de la musicologie universitaire), d'accueillir des questionnements aux frontières des sciences sociales (l'acoustique, l'architecture, les mathématiques, les sciences cognitives), tout en faisant bénéficier les étudiants de l'impressionnant catalogue de séminaires "hors parcours " proposés par des chercheurs de l'école sur les diverses aires culturelles et domaines de spécialité. Ce nouveau modèle de formation, que d'aucuns qualifient de «science indisciplinée de la musique $^{12}$ " et d'autres de "nouvelle musicologi ${ }^{13}$ ", attire un nombre toujours croissant d'étudiants et de doctorants venus de l'étranger, des grandes écoles et 
d'universités, aussi bien que des mondes professionnels de la musique. Il a nourri la création de la revue Transposition. Cette institutionnalisation d'un champ d'étude visant «la musique et les sciences sociales » n'empêche pas que se poursuivent, en d'autres lieux de l'École, des recherches, travaux et séminaires croisant de manière ponctuelle ou durable la musique par le biais de thématiques connexes (pratiques sociales, rituels, danses, formes spectaculaires, etc. ${ }^{14}$ ), sans que leurs auteurs ne mettent nécessairement en avant la spécificité de cet objet ${ }^{15}$. Plus largement, une réflexion sur la place de la musique dans les travaux et les recherches conduits à l'EHESS doit ainsi prendre en compte, outre certains lieux et moments de cristallisation, la permanence structurelle d'un mode d'existence diffus et, en somme, d'un foisonnement que ce numéro ne prétend aucunement épuiser.

5 C'est donc dans cette double tension - entre une croyance commune dans la « singularité EHESS » et une multiplicité de démarches de connaissance visant un objet "musique», dont certaines se trouvent aujourd'hui rassemblées sous des intitulés communs de formations, programmes de recherche et publications -, que se loge l'ambition de ce numéro hors-série. Au-delà d'un objectif de commémoration ou de célébration d'une communauté imaginée, nous présentons d'abord ici des textes qui font retour sur des recherches ayant investi l'espace de séminaires à l'EHESS. Jacques Cheyronnaud revient sur le séminaire tenu entre 1985 et 1993 au sein du DEA « Anthropologie sociale et Ethnologie » qui problématisait un objet « musique » avec les outils de l'anthropologie historique, à distance de l'approche qui prévalait jusqu'ici dans l'ethnomusicologie régionale de la France autant que des présupposés présidant à l'institutionnalisation d'un secteur des «musiques traditionnelles». Denis Laborde évoque le séminaire qu'il anime depuis une vingtaine d'années à l'École qui, dans une filiation revendiquée à celui de Jacques Cheyronnaud, a pris pour objets d'une investigation anthropologique l'histoire des collectes de chants populaires, l'improvisation sur timbre, les festivals de musiques du monde et, plus récemment, les liens entre musique, immigration et politiques urbaines. Les contributions de Michael Werner et Marc Chemillier proposent également un bilan de leurs séminaires. Le séminaire "Lieux et espaces de la musique", initié en 2002 par Michael Werner et Patrice Veit et auxquels se sont joints, par la suite, d'autres chercheurs (Denis Laborde, Karine Le Bail, Claire Guiu, Laura Jouve-Villard) a exploré pendant douze années des questionnements ayant trait à la spatialité, matérielle et symbolique, de l'activité musicale et à son inscription dans des lieux et des espaces sociaux, en faisant dialoguer des chercheurs et des acteurs du monde de la musique. Marc Chemillier, quant à lui, présente les enjeux de son séminaire "Modélisation des savoirs musicaux relevant de l'oralité " en développant les concepts de modélisation, d'expérimentation et de simulation et en les illustrant par des cas tirés d'une enquête de terrain à Madagascar et d'expérimentations menées autour du logiciel «Improtech». Laure Schnapper propose un bilan de son séminaire "Musicologie générale. Analyse et écoute comparatives", qui fait partie des enseignements de tronc commun des étudiants inscrits dans la spécialité « Musique » de l'EHESS. Fondé sur l'écoute et l'analyse des procédés formels, cet atelier amène l'auteure à insister sur une continuité de questionnements soulevés par des répertoires qui sont d'ordinaire traités de manière séparée par les historiens, ethnomusicologues et sociologues de la musique.

6 Outre ces contributions, qui permettent de faire ressortir la spécificité du dispositif du "séminaire» comme une composante importante de l'institution, nous avions également proposé aux auteurs sollicités de faire retour sur leurs itinéraires de 
recherche ou sur certains projets collectifs menés au sein de (ou en partenariat avec) l'EHESS. C'est cette option qu'ont retenue Nicolas Donin, Esteban Buch, Antoine Hennion et Philippe Le Guern. L'article de Nicolas Donin revient sur les recherches qu'il a menées, avec divers collaborateurs, autour de l'analyse des processus de composition d'œuvres de Philippe Leroux (Voi(rex) ou Apocalypsis), recherches qui ont permis de faire émerger un champ hybride de questionnements au croisement de la musicologie empirique, des sciences sociales et sciences cognitives. L'article d'Esteban Buch retrace son parcours comme étudiant à l'EHESS au début des années 1990, les séminaires qu'il a fréquentés et les travaux qui ont nourri sa compréhension du fait musical, de la sémiotique héritée du structuralisme au grand chantier des "lieux de mémoire » en passant par la déconstruction et la théorie critique, l'herméneutique et en débouchant sur une réflexion transversale sur l'écoute. À partir de cet exercice réflexif, l'auteur plaide pour une approche renouvelée de l'analyse musicale qui, une fois libérée du graphocentrisme et des hiérarchies communes établies entre les genres musicaux, peut s'intégrer aux protocoles de recherche des sciences sociales. Antoine Hennion revient, dans son texte, sur son parcours de recherche, placé sous le signe d'auteurs comme Michel de Certeau, Louis Marin, William James ou Étienne Souriau et interroge le rapport qu'entretient la sociologie de l'art à son objet. Enfin, le texte de Philippe Le Guern propose une mise en perspective de ses travaux et interroge les effets du virage numérique aussi bien sur les acteurs et dispositifs (musiciens, ingénieurs du son, échantillonneurs, home-studios, etc.) que sur les auditeurs.

$7 \mathrm{Si}$, pour la période récente, la plupart des personnes que nous avons sollicitées ont pu proposer un texte sur un séminaire ou sur leur parcours de recherche, nous avons dû procéder autrement pour des séminaires plus anciens, sur lesquels subsistent aujourd'hui peu de traces et des mémoires parfois contradictoires. Claudie MarcelDubois, qui eut, dès 1961-1962, une charge de conférences en ethnomusicologie générale à l'EPHE, dans le cadre de la direction d'études de Claude Lévi-Strauss, tout en assumant par ailleurs de nombreuses autres responsabilités ${ }^{16}$, est l'une de ces figures aujourd'hui méconnues des jeunes générations d'ethnomusicologues. En attendant d'autres travaux sur l'activité de cette chercheuse ${ }^{17}$, nous reproduisons ici un texte choisi sur la suggestion de Florence Gétreau (CNRS/IReMus), qui rappelle combien le domaine de l'organologie, central dans les trajectoires de nombreuses figures fondatrices de la musicologie comparée (comme Curt Sachs ou Erich von Hornbostel) et de l'ethnologie musicale (comme André Schaeffner ${ }^{18}$ ) soulève des questions cruciales pour l'étude des représentations sociales et culturelles de la musique. Une autre figure pionnière fut Françoise Escal, directrice d'études à l'EHESS à partir de 1989, qui noua au sein du Centre de Recherches sur les Arts et le Langage (CRAL) un dialogue avec des figures historiques du structuralisme et participa activement à une dynamique de réflexion sur la place de la musique au sein des sciences humaines et sociales ${ }^{19}$. Nous reproduisons ici un de ses textes, présenté par Esteban Buch, qui a le mérite d'évoquer aussi le rôle joué par la figure tutélaire de Roland Barthes dans les études sur les arts, à l'EHESS et bien au-delà. Le compositeur et musicologue François-Bernard Mâche, directeur d'études à l'EHESS entre 1993 et 1999, y proposa un programme de recherche consacré aux thèmes des universaux en musique, dont rend compte un texte issu d'une conférence prononcée en 1994 dans le cadre du colloque «La musique au regard des sciences humaines et des sciences sociales", que nous reproduisons ici avec une présentation de Márta Grabócz (professeure en musicologie à l'Université de Strasbourg). En complément, le témoignage d'une auditrice de son séminaire, Anne- 
Sylvie Barthel-Calvet (maitre de conférences à l'Université de Lorraine), permet de mieux situer cette pensée dans l'horizon des savoirs musicaux de l'époque et de faire apparaître le potentiel heuristique d'une démarche qui questionne le partage des rôles entre savant et créateur. Enfin, le témoignage de François Picard sur le séminaire que Tran Van Khê consacra à la thématique "Les chants alternés en Asie du Sud-Est » au milieu des années 1980, rappelle combien ont pu compter, notamment pour le domaine de l'ethnomusicologie, l'édification de réseaux et groupes de réflexion regroupant des spécialistes d'une même aire culturelle, chercheurs et praticiens de la musique. Sans épuiser les discussions qui ont pu se dérouler dans chacun des séminaires animés par ces pionniers, nous espérons que ces quelques textes et témoignages rassemblés dans la rubrique "Réminiscences " susciteront l'envie de nos lecteurs de se replonger dans ces travaux, que ce soit pour y puiser des outils ou pour poursuivre une réflexion historiographique les relations entre musicologie et sciences humaines.

8 Au fil de la préparation de ce numéro a, enfin, germé l'idée, venue d'Elsa Rieu, d'une troisième rubrique: «L'EHESS vue d'ailleurs ». Nous avons sollicité des chercheurs étrangers, qui avaient fréquenté l'École en tant qu'étudiants ou enseignants invités, pour des témoignages rétrospectifs sur ce temps passé à l'EHESS. Aux contributions de William Weber et de Jann Pasler, deux chercheurs américains dont le parcours scientifique a été étroitement lié à des séminaires et réflexions de chercheurs de l'École, répondent celles de trois jeunes chercheuses arrivées comme doctorantes à l'EHESS. Venues d'Allemagne, d'Argentine, d'Italie, et depuis recrutées dans des départements de musicologie, dans leurs pays d'origine (Gesa zur Nieden, Marina Cañardo) ou en France (Marta Amico), elles se livrent depuis à la délicate tâche de « traduire » les enseignements acquis à l'EHESS en ces lieux, pour des publics souvent plus jeunes et plus nombreux. Leurs contributions témoignent d'un aspect commun à beaucoup d'autres contributeurs de ce numéro, qui est celui des allers-retours pratiqués entre différentes traditions nationales des sciences sociales ou des sciences de la culture, sources de réflexivité et de regards croisés.

À partir de ces contributions, le lecteur pourra ainsi trouver matière à questionner sous de multiples angles le postulat d'une "exception EHESS»: tant au regard des renouvellements survenus dans le champ des sciences de la musique et des sciences sociales que des dynamiques d'internationalisation des productions scientifiques et des transformations contemporaines des mondes de la musique. Il pourra aussi tâcher d'esquisser des recoupements dans la foisonnante pluralité d'objets et de méthodes évoqués dans ces diverses contributions, mais aussi prendre la mesure des différences et contradictions qu'elles dessinent, sans chercher à les subsumer sous le modèle d'une discipline de référence. Peut-être pourra-t-il voir dans cette pluralité l'indice d'une fragilité épistémique ou, tout aussi bien, d'une vertu heuristique de la musique à bousculer les partages établis entre divers domaines des sciences. Sans préjuger ici de l'avis de nos lecteurs, nous souhaitons en tout cas que tous et toutes puissent puiser dans les textes qui vont suivre des outils pour bâtir leurs propres chemins de réflexion. $\mathrm{Au}$ terme de cette aventure éditoriale, il ne nous reste ainsi que l'agréable tâche de remercier les auteurses pour leurs contributions et leur patience, ainsi que les personnes qui ont apporté leur concours pour la traduction (Michelle Biget-Mainfroy et Elsa Broclain) et le travail éditorial (Étienne Jardin). 


\section{BIBLIOGRAPHIE}

Cahiers d'études africaines 168 (2002) : « Musiques du monde » sous la direction de Bob W. WHITE.

Cahiers d'études africaines 216 (2014) : « Musiques dans l'“atlantique noir" ».

Filigrane 11 (2010) : « New Musicology. Perspectives critiques » sous la direction de Makis SOLOMOS et Márta GRABÓcZ.

L'Homme 158-159 (2001) : « Jazz et anthropologie » sous la direction de Jean JAMIN et Patrick WILLIAMS.

L'Homme 171-172 (2004) : « Musique et anthropologie ».

L'Homme 177-178 (2006) : « Chanter, musiquer, écouter ».

L'Homme 215-216 (2015) : « Connaît-on la chanson?»

Revue de musicologie 103/2 (2017) : " Un siècle de musicologie en France. Histoire intellectuelle de la Revue de musicologie. Vol 1 » sous la direction d'Yves BALMER et Hervé LACOMBE.

Revue de musicologie 104/1 (2018) : « Un siècle de musicologie en France. Histoire intellectuelle de la Revue de musicologie. Vol 2 » sous la direction d'Yves BALMER et Hervé LACOMBE.

Revue d'histoire des sciences humaines 14 (2006) : « Musique et sciences humaines, rendez-vous manqués? » sous la direction de Rémy CAMPOS, Nicolas DONIN et Frédéric KECK. Disponible en ligne : https://www.cairn.info/revue-histoire-des-sciences-humaines-2006-1.htm (consulté le 27 avril 2018).

CAMPOS, Rémy, DONIN, Nicolas et KECK, Frédéric, « Musique, musicologie, sciences humaines : sociabilités intellectuelles, engagements esthétiques et malentendus disciplinaires (1870-1970) », Revue d'histoire des sciences humaines 14 (2006), p. 3-17.

CHIMÈNES, Myriam, « Musicologie et histoire. Frontière ou "no man's land" entre deux disciplines? », Revue de musicologie 84-1 (1998), p. 67-78.

ESCAL, Françoise, Espaces sociaux, espaces musicaux, Paris, Payot, 1979.

ESCAL, Françoise et IMBERTY, Michel (éds), La musique au regard des sciences humaines et des sciences sociales, 2 Vol., Paris, L'Harmattan, 1997.

ESCAL, Françoise, Le concert : enjeux, fonctions, modalités, Paris, L'Harmattan, 2000.

FABIANI, Jean-Louis, « À quoi sert la notion de discipline? ", Qu'est-ce qu'une discipline, sous la direction de Jean BOUTIER, Jean-Claude PASSERON et Jacques REVEL, Paris, Éditions de l'EHESS, 2006, p. 11-34.

FORNEL, Michel de et QUÉRÉ, Louis (éds), La logique des situations. Nouveaux regards sur l'écologie des activités sociales, Paris, Éditions de l'EHESS, 1999.

HERZFELD, Michael, Cultural intimacy: Social poetics in the nation-state, New York, Routledge, 2014.

LABORDE, Denis, "Pour une science indisciplinée de la musique », in BACHIR-LOOPUYT, Talia, IGLESIAS, Sara, LANGENBRUCH, Anna et NIEDEN, Gesa zur (éds), Musiques, contextes, savoirs. Perspectives interdisciplinaires sur la musique/Musik, Kontext, Wissenschaft. Interdisziplinäre Forschung zu Musik, Frankfurt am Main, Peter Lang, 2012, p. 25 - 33.

NATTIEZ, Jean-Jacques, « Musicologie historique, ethnomusicologie, analyse : Une musicologie générale est-elle possible ? », Musicae Scientiae 14/2 (2010), p. 333-355. 
PASSERON, Jean Claude et REVEL, Jacques (éds), Penser par cas, Paris, Éditions de l'EHESS, 2005. PECQUEUX, Anthony et ROUEFF Olivier (éds), Écologie sociale de l'oreille. Enquêtes sur l'expérience musicale, Paris, Éditions de l'EHESS, 2009.

PEDLER, Emmanuel et CHEYRONNAUD, Jacques (éds), Théories ordinaires, Paris, Éditions de l'EHESS, 2013.

RAVET, Hyacinthe, L'année sociologique 60/2 (2010) : « Sociologies de la musique », p. 271-303.

SAHLINS, Marshall, Des îles dans l'histoire, Gallimard, Le Seuil, 1989.

SCHAEFFNER, André, Origine des instruments de musique. Introduction ethnologique à l'histoire des instruments de musique, Paris, Éditions de l'EHESS, 1994 [1re éd. 1968].

TRAVERSIER, Mélanie, « Histoire sociale et musicologie : un tournant historiographique », Revue d'histoire moderne et contemporaine 57/2 (2010), p. 190-201.

WERNER, Michael et ZIMMERMANN, Bénédicte, « Penser l'histoire croisée : entre empirie et réflexivité », Annales. Histoire, sciences sociales 58/1 (2003), p. 7-36.

\section{NOTES}

1. Ceux de «grands événements» $(1918,1968)$ tout comme d'épisodes de l'histoire des sciences qui sont parfois l'occasion de vastes entreprises historiographiques. Voir en particulier, pour la musicologie, l'important travail effectué sous la coordination d'Yves Balmer et Hervé Lacombe à l'occasion du centenaire de la Société française de musicologie, donnant lieu à deux imposants numéros de la Revue de musicologie en 2017 et 2018 (103/2 et 104/1).

2. Nous tenons ici à remercier Elsa Rieu, qui a activement accompagné la préparation de ce numéro et les premières relectures, avant de se retirer pour des raisons de santé.

3. En 2001 est créée la formation doctorale "Musique, Histoire, Société ", qui accueille des étudiants à partir du DEA. En 2004, le système de Bologne conduit à la création du Master « Musique ", également en partenariat avec le CNSMDP, l'EPHE, l'ENS et l'IRCAM.

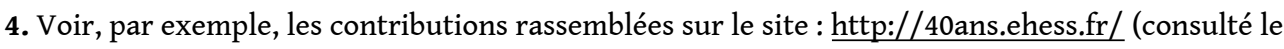
27 avril 2018).

5. Le colloque "Arts, littérature et sciences sociales » qui s'est tenu à l'EHESS les 19 et 20 juin 2015 a en particulier fait ressortir l'importance des héritages de Roland Barthes et Pierre Bourdieu. Voir les vidéos en ligne de ce colloque: https://www.canal-u.tv/producteurs/ehess/ 40_ans_de_1_ehess/arts_litterature_et_sciences_sociales (consulté le 27 avril 2018).

6. Que l'on peut rapprocher de la notion d'intimité sociale forgée par Michael Herzfeld. Voir HERZFELD, Michael, Cultural intimacy: Social poetics in the nation-state, New York, Routledge, 2014.

7. Il revient peut-être à la "pensée par cas» la palme de l'outil le plus cité par les jeunes chercheurs, avec les paradigmes de «l'histoire croisée » et de l'analyse située. Voir: PASSERON, Jean-Claude et REVEL, Jacques (éds), Penser par cas, Paris, Éditions de l'EHESS, 2005 ; WERNER, Michael et ZIMMERMANN, Bénédicte, «Penser l'histoire croisée : entre empirie et réflexivité ", Annales. Histoire, sciences sociales 58/1 (2003), p. 7-36 ; FORNEL, Michel de et QUÉRÉ, Louis (éds), La Logique des situations. Nouveaux regards sur l'écologie des activités sociales, Paris, Éditions de l'EHESS, 1999. Les diverses collections des Éditions de l'EHESS ont joué un rôle important dans l'édification de questionnements transversaux marquant aujourd'hui ce que l'on pourrait appeler une « culture EHESS ». Voir en particulier : https://journals.openedition.org/enquete/ (consulté le 27 avril 2018). Sur la musique, outre les travaux des contributeurs de ce numéro, voir, aux Éditions de l'EHESS : L'Homme 158-159 (2001) : « Jazz et anthropologie » sous la direction de Jean JAMIN et Patrick WILLIAMS ; L'Homme 171-172 (2004) : « Musique et anthropologie »; L'Homme 
177-178 (2006) : "Chanter, musiquer, écouter»; L'Homme 215-216 (2015): "Connaît-on la chanson? »; Cahiers d'études africaine 168 (2002) : « Musiques du monde » sous la direction de Bob W. WHITE ; Cahiers d'études africaine 216 (2014) : « Musiques dans l'“atlantique noir" "; PECQUEUX Anthony et ROUEFF Olivier (éds), Écologie sociale de l'oreille. Enquêtes sur l'expérience musicale, Paris, Éditions de l'EHESS, 2009; PEDLER, Emmanuel et CHEYRONNAUD, Jacques (éds), Théories ordinaires, Paris, Éditions de l'EHESS, 2013.

8. Nous reprenons ici une distinction opérée par Jean-Louis Fabiani entre discipline, savoir et démarches de connaissance dans FABIANI, Jean-Louis, "À quoi sert la notion de discipline?", Qu'est-ce qu'une discipline, sous la direction de Jean BOUTIER, JeanClaude PASSERON et Jacques REVEL, Paris, Éditions de l'EHESS, 2006, p. 11-34.

9. Voir à ce propos, outre le double numéro déjà cité du centenaire de la Revue de musicologie : CHIMÈNES, Myriam, «Musicologie et histoire. Frontière ou "no man's land" entre deux disciplines ? ", Revue de musicologie 84-1 (1998), p. 67-78 ; TRAVERSIER, Mélanie, « Histoire sociale et musicologie : un tournant historiographique ", Revue d'histoire moderne et contemporaine 57/2 (2010), p. 190-201; Revue d'histoire des sciences humaines 14 (2006): «Musique et sciences humaines, rendez-vous manqués ?» sous la direction de Rémy CAMPOS, Nicolas DONIN et Frédéric KECK, disponible en ligne: https://www.cairn.info/revue-histoire-des-scienceshumaines-2006-1.htm (consulté le 27 avril 2018).

10. Voir RAVET, Hyacinthe, L'année sociologique 60/2 (2010): "Sociologies de la musique", p. 271-303.

11. En particulier au sein du Centre de Recherches sur les Arts et le Langage (CRAL), du Centre de Recherches Interdisciplinaires sur l'Allemagne (CRIA), aujourd'hui rebaptisé Centre Georg Simmel, et du groupe travaillant à une anthropologie du jazz autour de Jean Jamin (LAS) et Patrick Williams (LAUM).

12. LABORDE, Denis, « Pour une science indisciplinée de la musique », in BACHIR-LOOPUYT, Talia, IGLESIAS, Sara, LANGENBRUCH, Anna et NIEDEN, Gesa zur (éds), Musiques, contextes, savoirs. Perspectives interdisciplinaires sur la musique / Musik, Kontext, Wissenschaft. Interdisziplinäre Forschung zu Musik, Frankfurt am Main, Peter Lang, 2012, p. 25 - 33.

13. Voir, dans ce numéro, la contribution d'Esteban Buch et sa référence à l'ouvrage de ESCAL, Françoise, Espaces sociaux, espaces musicaux, Paris, Payot, 1979. Dans le contexte anglophone, la notion de nouvelle musicologie fait référence à un ensemble de courants marqués par les apports des cultural studies, des études féministes et des études postcoloniales notamment. Voir, pour une réinterprétation de ces travaux en France, SOLOMOS, Makis et Márta, GRABÓCZ (éds.), Filigrane 11 (2010) : « New Musicology. Perspectives critiques».

14. Voir en particulier les compte-rendus de séminaires d'Elizabeth Claire (histoire culturelle de la danse), de Jacques Cheyronnaud et Emmanuel Pedler (la forme spectacle).

15. Le cas de ce que certains appellent aujourd'hui, dans le langage autochtone, "l'école de Marseille" (une génération de chercheurs formés à l'EHESS Marseille auprès de Jacques Cheyronnaud, Jean-Louis Fabiani et Emmanuel Pedler) est particulièrement exemplaire d'une réflexion collective qui, sans mettre en exergue une spécialisation sur "la musique ", s'est pourtant constamment nourrie de réflexions sur des terrains musicaux. Voir notamment PECQUEUX, Anthony et ROUEFF Olivier (éds), Écologie sociale de l'oreille. Enquêtes sur l'expérience musicale, Paris, Éditions de l'EHESS, 2009. Voir également les travaux de Pierre-Michel Menger, Jean Jamin, Patrick Williams, Elizabeth Claire, Daniel Fabre et bien d'autres.

16. Comme directrice du département d'ethnomusicologie du MNATP, comme membre du Conseil d'administration de la Société française de musicologie (de 1973 à 1988), comme membre puis présidente (1967-1970) de la Section Anthropologie-Ethnologie-Préhistoire du CNRS, comme vice-présidente (1973-1978) et présidente (1974-1987) de la Société d'ethnologie française. 
17. Signalons ici le projet en cours mené par François Gasnault et Marie Barbara Le Gonidec sur l'histoire de l'ethnomusicologie en France (LAHIC/IIAC, programme SAHIEF), qui a donné lieu à un cycle de conférences en 2015, avec la collaboration du regretté Daniel Fabre: http:// www.cirief.fr/le-cirief/manifestations/88-cycle-de-conferences-iiac-sources-archives-ethistoire-institutionnelle-de-l-ethnomusicologie-de-la-france (consulté le 27 avril 2018).

18. Dont le traité Origine des instruments de musique. Introduction ethnologique à l'histoire des instruments de musique, paru en 1936, a été réédité aux Éditions de l'EHESS en 1968.

19. Voir, en particulier, de cette auteure : ESCAL, Françoise. Le concert: enjeux, fonctions, modalités, Paris, L'Harmattan, 2000 ; ESCAL, Françoise et IMBERTY, Michel (éds), La Musique au regard des sciences humaines et des sciences sociales, 2 Vols., Paris, L'Harmattan, 1997. 